\title{
Modelo de centro de distribución verde: amigabilidad con el medio ambiente y eficiencia operacional usando un enfoque de procesos y un metaheurístico de búsqueda tabú
}

\author{
Rodrigo Andrés Gómez Montoya* \\ Alexander Alberto Correa Espinal ${ }^{* *}$ \\ José Daniel Hernández Vahos ${ }^{* * *}$
}

Recibido: 16/07/2015 - Aceptado: 23/06/2016

DOI: $10.22395 /$ rium.v16n31a10

\section{Resumen}

Este artículo tiene como objetivo desarrollar y validar un modelo de centro de distribución (CEDI) verde, que se conforma de tres componentes: un módulo de gestión, un sistema de administración de almacenes (WMS-Warehouse Management System) y un metaheurístico. Como resultado de la validación del modelo en un CEDI de una empresa mediana de alimentos, se obtuvo una reducción de las emisiones de $\mathrm{CO}_{2}$ equivalente de $731 \mathrm{~kg} / \mathrm{mes}$ o un $37 \%$ de las emisiones en la preparación de pedidos. Además, se desarrolló el modelamiento de un metaheurístico de búsqueda tabú para resolver el problema de ruteo en la preparación de pedidos, lo que permitió incrementar la eficiencia de la operación en un 18,83\%, así como obtener una reducción de $198 \mathrm{~kg}$ de $\mathrm{CO}_{2}$ equivalente por mes. Por lo tanto, el modelo CEDI verde, simultáneamente, aumentó la eficiencia y redujo la cantidad de kilogramos de $\mathrm{CO}_{2}$, utilizando un enfoque que no había sido abordado en la literatura para este proceso logístico.

Palabras clave: centro de distribución; emisiones $\mathrm{CO}_{2}$; logística verde; medio ambiente; metaheurísticos; tecnologías de la información y comunicaciones.

* Ph.D. en Ingeniería: Industria y Organizaciones. Profesor e investigador, Facultad de Ciencias Agrarias, Politécnico Colombiano Jaime Isaza Cadavid e Investigador Asociado Grupo Modelamiento en Gestión de las Operaciones (GIMGO), Facultad de Minas, Universidad Nacional de Colombia, Sede Medellín. ragomez@ elpoli.edu.co

** Ph.D. en Estadística e Investigación Operativa. Profesor Asociado, Departamento Ingeniería de la Organización, Facultad de Minas, Universidad Nacional de Colombia, Sede Medellín. alcorrea@unal.edu.co

*** Investigador Junior Grupo Modelamiento en Gestión de las Operaciones (GIMGO), Facultad de Minas, Universidad Nacional de Colombia, Sede Medellín.jdhernandezv@unal.edu.co 


\title{
Green distribution center model: environmentally friendly and operational efficiency using a process approach and a taboo search metaheuristics
}

\begin{abstract}
This article is intended to develop and validate a green distribution center model consisting of three components: a management module; a warehouse management system (WMS); and a metaheuristics. As a result of the validation of the model at a Distribution Center of a medium-size food company, a reduction of $\mathrm{CO}_{2}$ emissions was achieved, equivalent to $731 \mathrm{~kg} / \mathrm{month}$ or $37 \%$ of issues for preparation of orders. Additionally, the modeling of a Taboo Search metaheuristics was developed to resolve the routing problem for the preparation of orders; this allowed increasing the operation efficiency in about $18.83 \%$ and to obtain a reduction of $198 \mathrm{~kg}$ of equivalent $\mathrm{CO}_{2}$ per month. Therefore, the green distribution center model, simultaneously, increased the efficiency and reduced the amount of kilograms of $\mathrm{CO}_{2}$, using an approach that had not been addressed in literature for this logistic process.
\end{abstract}

Keywords: distribution center; $\mathrm{CO}_{2}$ emissions; green logistics; environment; metaheuristics; information and communication technologies.

\section{Modelo de centro de distribuição verde: amigabilidade com o meio ambiente e eficiência operacional com uma abordagem de processos e uma meta-heurística de pesquisa tabu}

\begin{abstract}
Resumo
Este artigo tem como objetivo desenvolver e validar um modelo de centro de distribuição (Cedi) verde formado por três componentes: um módulo de gestão, um sistema de administração de armazéns (WMS-Warehouse Management System) e uma meta-heurística. Como resultado da validação do modelo num Cedi de uma empresa média de alimentos, obteve-se uma redução das emissões de $\mathrm{CO}_{2}$ equivalente de $731 \mathrm{~kg} / \mathrm{mês}$ ou $37 \%$ das emissões na preparação de pedidos. Além disso, desenvolveu-se o modelamento de uma meta-heurística de pesquisa tabu para resolver o problema de roteamento na preparação de pedidos, o que permitiu aumentar a eficiência da operação em 18,83\%, bem como obter uma redução de 198 $\mathrm{kg}$ de $\mathrm{CO}_{2}$ equivalente por mês. Portanto, o modelo de Cedi verde, ao mesmo tempo, aumentou a eficiência e reduziu a quantidade de quilograma de $\mathrm{CO}_{2}$ utilizando uma nova abordagem na literatura para esse processo logístico.
\end{abstract}

Palavras-chave: centro de distribuição; emissões de $\mathrm{CO}_{2}$; logística verde; meio ambiente; meta-heurística; tecnologias de da informação e comunicação. 


\section{INTRODUCCIÓN}

El Centro de Distribución (CEDI) permite recibir y almacenar los productos que provienen de una fábrica o proveedor, así como atender los requerimientos de los clientes en las condiciones pactadas [1, 2]. Inclusive, el CEDI puede considerarse un proceso que integra a los eslabones de la cadena de suministro, ya que permite recibir, almacenar y despachar los pedidos en la cadena de suministro [3]. El CEDI pueden ser clasificado como: i) manuales y automatizados, ii) hombre a producto y producto a hombre y iii) sistematizados con tecnologías de información y comunicaciones (TIC) y sin sistematizar $[3,4,5]$.

En el CEDI la preparación de pedidos consiste en recoger los productos solicitados en las órdenes de los clientes, con el fin de satisfacer adecuadamente sus requerimientos en el menor tiempo y costo posible [6]. Bartholdi y Hackman [3] indican que esta operación suele ser la más importante del CEDI, ya que tiene un impacto directo en la satisfacción de los clientes y representa alrededor del 55\% de los costos de este proceso logístico. Por el motivo expuesto, en la literatura científica del CEDI han sido formulados problemas de asignación de posiciones de almacenamiento, conformación de lotes y ruteo en la preparación de pedidos buscando que esta operación sea eficiente y eficaz $[7,8]$.

Una búsqueda exhaustiva en la literatura científica fue realizada para establecer si se han publicado artículos que modelen enfoques de CEDI o gestión de almacenes verdes. De la revisión realizada, no se detectaron artículos que aborden el enfoque de CEDI verdes, producción limpia u otras prácticas que permitan una operación amigable con el medio ambiente, que generen la cantidad mínima de $\mathrm{CO}_{2}$ posible y que promuevan el uso adecuado de los recursos. Debe resaltarse que la logística verde consiste en implementar políticas, prácticas y planes ambientales en los sistemas logísticos [9]. Lo que se identificó en la literatura fueron artículos de logística verde, los cuales incluyen la gestión de almacenamiento y CEDI como un proceso implícito dentro de la logística. En la literatura científica se identificaron artículos de autores tales como: Ubeda, Arcelus y Faulin (2011), Dekker, Bloemhof y Mallidis (2012), Martinsen y Björklund (2012), Zhang y Zhao (2012) y Zhao y Gu,(2014), Harris, Mumford y Naim (2014) y Zhang, Lee, Chan, Choy, y Wu, (2015) que implementaron o estructuraron modelos de gestión de la logística verde, incluyendo prácticas, políticas e indicadores para su medición $[10,11,12,13,14,15,16]$. Adicionalmente, autores como: Lai y Wong (2012), Pishvaee, Torabi y Razmi (2012), Dobers, Klukas, Lammers, Laux, Mauer y Schneider (2013) y Ćirović, Pamučar y Božanić (2014) desarrollaron modelos cuantitativos para aumentar la eficiencia operacional y reducir las emisiones del $\mathrm{CO}_{2}$, a través del diseño o mejoramiento de los procesos logísticos [17, 18, 19, 20]. Finalmente, debe resaltarse que en la literatura no se detectaron artículos de logística verde que utilicen 
TIC, tales como WMS, ERP(por sus siglas en inglés, Enterprise Resource Planning), entre otros, para implementar prácticas que reduzcan la cantidad de $\mathrm{CO}_{2}$ o consumo innecesario de papel.

Debido a los hallazgos obtenidos, el presente artículo tiene como objetivo desarrollar y validar un modelo de CEDI verde soportado en la implementación de prácticas de gestión, uso de un WMS (TIC) y un metaheurístico que permitan apoyar decisiones de diseño o mejoramiento de sus operaciones, con el fin de operar efectivamente el proceso y reducir la cantidad de $\mathrm{kg}$ de $\mathrm{CO}_{2}$, lo cual promueve un enfoque de amigabilidad con el medio ambiente. De esta manera, se pretende contribuir al avance del conocimiento en el área de investigación de la logística verde, ya que este artículo sería uno de los primeros en abordar el enfoque de CEDI Verde en el ámbito científico.

Las demás secciones del presente artículo y su contenido son: En la segunda sección, se desarrolla la estructura metodológica del modelo de CEDI. En la tercera sección, se planea, implementa y discuten los resultados de un caso de estudio para el modelo de CEDI verde. Finalmente, en la cuarta sección, se presentan las conclusiones, hallazgos y aportes al conocimiento obtenidos.

\section{METODOLOGÍA: MODELO DE CEDI VERDE SOPORTADO EN PRÁCTICAS DE GESTIÓN, WMS Y MODELAMIENTO CUANTITATIVO CON METAHEURÍSTICOS}

Esta sección tiene como objetivo desarrollar un modelo de CEDI verde soportado en prácticas de gestión, WMS (TIC) y un metaheurístico (modelamiento cuantitativo). Algunos principios y enfoques de los modelos de Dekker et al. (2012), Harris et al.,(2014) y Zhang et al.,(2015) fueron utilizados de referencia para desarrollar la presente propuesta metodológica $[11,20]$. A continuación, se representa la estructura del modelo de CEDI verde propuesto.

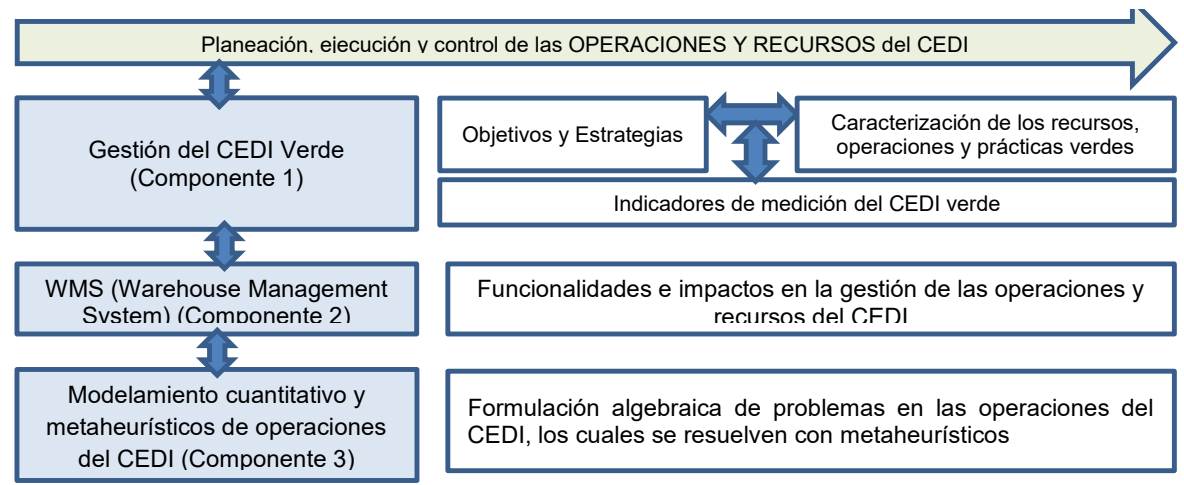

Figura 1: estructura metodológica del modelo del CEDI verde.

Fuente: elaboración propia. 
La estructura del modelo CEDI verde (figura 1) está conformada por tres componentes, los cuales impactan transversalmente la planeación, ejecución y control de las operaciones y recursos de este proceso logístico (figura 1). El primer componente denominado gestión incluye los objetivos y estrategias del CEDI, la caracterización de los procesos e indicadores del modelo CEDI Verde. El segundo componente es un $W M S$ que sistematiza las operaciones y recursos del CEDI, lo cual elimina el uso del papel y contribuye al aumento de la efectividad. Por último, el tercer componente permite el modelamiento cuantitativo de problemas de asignación en el CEDI. Debe resaltarse que los componentes del modelo están sincronizados, lo cual busca generar un impacto integral al desarrollo del CEDI. A continuación, se desarrollan los componentes descritos.

\subsection{Componente 1: gestión del CEDI Verde}

Este componente establece la estructura de gestión para el CEDI verde considerando tres subcomponentes denominados: i) objetivos y estrategias, ii) caracterización de los recursos, operaciones y prácticas verdes, iii) indicadores del CEDI verde. A continuación, se desarrollan cada uno de los subcomponentes:

\subsubsection{Objetivos y estrategias del CEDI verde}

En este subcomponente se establecen objetivos para el CEDI verde, los cuales deben ser alineados con la planeación estratégica del proceso y el sistema logístico de compañía. Posteriormente para cada objetivo formulado se diseña, por lo menos, una estrategia que establece las actividades para su implementación.

\subsubsection{Caracterización de los recursos, operaciones y prácticas verdes en el CEDI}

En este subcomponente se utiliza una metodología adaptada del Sipoc (Supplier, Input, Processes, Output, Costumer) que permite caracterizar las operaciones del CEDI, incluyendo sus flujos de información, productos, equipos y personal, y establecer los recursos que suelen tener un impacto directo en el medio ambiente. En la caracterización, también se incluyen mediciones del CEDI verde relacionadas con las operaciones y recursos tales como energía (kwh), kg de papel, litros de gas consumidos por los equipos de manejo de materiales, agua (lt), entre otros. A continuación, se presenta esta caracterización: 
Tabla 1: Sipoc adaptado para el modelo de CEDI verde.

\begin{tabular}{|c|c|c|c|c|c|}
\hline Operación & & \multicolumn{3}{|c|}{ Alcance } & \multirow[b]{3}{*}{ Recursos } \\
\hline Objetivo & \multicolumn{4}{|c|}{ Objetivos relacionado con CEDI verde } & \\
\hline $\begin{array}{l}\text { Proveedor } \\
\text { (Suppliers) }\end{array}$ & Entradas (Inputs) & $\begin{array}{l}\text { Actividades } \\
\text { (Processes) }\end{array}$ & $\begin{array}{l}\text { Salidas } \\
\text { (Outputs) }\end{array}$ & $\begin{array}{c}\text { Clientes } \\
\text { (Costumers) }\end{array}$ & \\
\hline \multicolumn{6}{|c|}{ Prácticas verdes en la operación } \\
\hline \multicolumn{6}{|c|}{ Recursos (Equipo de manejo de materiales, personal, infraestructura, WMS o TIC, Otros recursos) } \\
\hline \multicolumn{6}{|c|}{ Indicadores del CEDI verde } \\
\hline \multicolumn{3}{|c|}{ Descripción del indicador } & \multicolumn{3}{|c|}{ Actual } \\
\hline
\end{tabular}

Fuente: elaboración propia

\subsubsection{Indicadores de medición del CEDI verde}

Este subcomponente permite medir el cumplimiento de los objetivos y el impacto de las prácticas verdes en el CEDI. A continuación, se presentan algunos indicadores verdes que pueden implementarse en el modelo propuesto (tabla 2). Por lo tanto, se pueden incluir otros, según las características del CEDI que se modele.

Tabla 2: indicadores CEDI verde.

\begin{tabular}{|l|l|}
\hline \multicolumn{1}{|c|}{ Indicador } & \multicolumn{1}{c|}{ Descripción } \\
\hline $\begin{array}{l}\text { Emisiones } \mathrm{CO}_{2} \text { de operaciones especificas o el CEDI } \\
\text { global medidas en kg }\end{array}$ & $\begin{array}{l}\text { Permite medir la cantidad } \mathrm{CO}_{2} \text { del CEDI u } \\
\text { operaciones particulares de éste, medido en } \mathrm{kg} . \\
\text { Esta medición puede ser realizada antes y des- } \\
\text { pués de implementar el modelo de CEDI verde. }\end{array}$ \\
\hline $\begin{array}{l}\text { Cantidad de papel consumido en el CEDI medido en } \\
\text { kilogramos }\end{array}$ & $\begin{array}{l}\text { Este indicador mide la cantidad de toneladas } \\
\text { de papel que se utilizan de manera global en } \\
\text { el CEDI u operaciones particulares de éste. }\end{array}$ \\
\hline $\begin{array}{l}\text { Cantidad de material de empaque y embalaje reutilizado } \\
\text { medido en unidades o kilogramos }\end{array}$ & $\begin{array}{l}\text { Este indicador es utilizado para medir la can- } \\
\text { tidad de material de empaque y embalaje que } \\
\text { es reutilizado en actividades del CEDI, con el } \\
\text { fin de generar un impacto positivo en el medio } \\
\text { ambiente. }\end{array}$ \\
\hline
\end{tabular}




\subsection{WMS (Warehouse Management System) como componente del modelo del CEDI verde}

Este segundo componente de la metodología consiste en el diseño, implementación y mantenimiento de un WMS (Warehouse Management System) o sistema de administración de almacenes, que permite sistematizar con tecnologías de la información y comunicaciones (TIC) las operaciones y recursos de este proceso logístico $[4,19]$. Las dos justificaciones para implementar un $W M S$ en el modelo CEDI verde son:

- Esta TIC apoya la planeación y control de las operaciones y recursos del CEDI. Por lo tanto, se asignan y gestionan efectivamente las operaciones del CEDI eliminando reprocesos y promoviendo adecuadamente el uso de los recursos en este proceso logístico.

- En el $W M S$ se modelan y sistematizan las operaciones con métodos que utilicen eficientemente los recursos, lo cual debe permitir la reducción de las emisiones de $\mathrm{CO}_{2}$, el uso de papel y otros recursos. Por lo tanto, el WMS busca establecer un enfoque paperless o CEDI sin papeles.

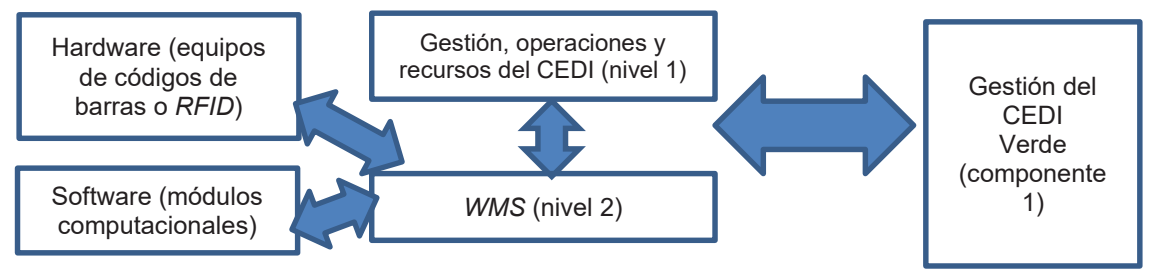

Figura 2: Estructura del WMS dentro del modelo de CEDI verde.

Fuente: elaboración propia

En el primer nivel de la estructura (figura 2), se encuentra la gestión (planeación, ejecución y control) de las operaciones y recursos del CEDI bajo modelamiento. En el segundo nivel, se encuentra el WMS que sistematiza con las TIC, las políticas, objetivos, operaciones y recursos caracterizados del CEDI. Finalmente, debe indicarse que el $W M S$ descrito es un desarrollo propio del grupo de investigación GIMGO de la Universidad Nacional de Colombia, Sede Medellín.

\subsection{Modelamiento cuantitativo de operaciones del CEDI con metaheurísticos (componente 3)}

Este tercer componente del modelo de CEDI Verde permite formular problemas $\mathrm{NP}$ hard de asignación en las operaciones del CEDI que se resuelven con metaheurísticos, con el fin de obtener las mejores soluciones posibles respecto a eficiencia, tiempos, costos o distancias. Por lo tanto, este componente del CEDI verde no solo pretende obtener las mejores condiciones operacionales, sino también aumentar el aprovechamiento de los recursos (electricidad, gas, papel, entre otros) utilizados en el CEDI, lo cual genera un impacto positivo en el medio ambiente. 
En el presente modelo de CEDI verde para obtener soluciones eficientes (menor tiempo o distancia posible) se analiza un problema de ruteo de la preparación de pedidos, que considera: i) múltiples productos de diferentes referencias, ii) $m$ posiciones de almacenamiento (diferentes niveles de altura) y iii) $K$ equipos heterogéneos (montacargas y transpaletas) de diferentes capacidades de carga, velocidad y elevación. El problema que se formula se resuelve con búsqueda tabú, un algoritmo genético u otros metaheurísticos [5, 8]. A continuación, se modela algebraicamente el problema planteado (figura 3):

\section{Índices y conjuntos}

$\begin{array}{ll}h, j \in L & \text { Posiciones de almacenamiento } \\ \mathrm{g} \in \mathrm{L} & \text { Subconjunto de posiciones de almacenamiento } \\ k \in K & \text { Equipo de manejo de materiales (EMM) específico } \\ \mathrm{r} \in \mathrm{R} & \text { Rutas de preparación de pedidos } \\ \mathrm{i} \in \mathrm{I} & \text { Producto } i \text { perteneciente a una lista de pedidos o una orden individual }\end{array}$

\begin{tabular}{|ll}
\hline \multicolumn{1}{c}{ Parámetros } \\
\hline$d^{h j}$ & Distancia entre dos posiciones de almacenamiento $h y j$ \\
$\mathrm{~V}_{\mathrm{k}}$ & Velocidad de viaje ( $\mathrm{m} / \mathrm{s})$ de cada EMM $k K$ \\
$t b e_{k i j}:$ & Tiempo para buscar, extraer y manipular un producto il desde la posición i a la j usando un EMM $\mathrm{kK}$ \\
$b_{i}$ & Cantidad de producto $i$ a ser recogido \\
$P_{i}$ & Disponibilidad de inventario de producto $i$ \\
$P_{i j}$ & Cantidad de inventario del producto $i$ almacenado en la posición $j$ \\
$L C_{i}:$ & Capacidad de carga utilizada por el producto $i$ \\
$A L C_{k}:$ & Capacidad de carga disponible del EMM $k K$
\end{tabular}

\begin{tabular}{|c|} 
Variables de decisión \\
$S_{h j}^{r}=\left\{\begin{array}{l}1, \text { Si la posición de almacenamiento } j \text { es visitada inmediatamente después de la posición } h \text { en una ruta } r \in \mathrm{R} \\
0, \text { otro caso }\end{array}\right.$ \\
$X_{i}^{r}=\left\{\begin{array}{l}1, \text { Si el producto } i \text { es asignado a la ruta } r \in R \\
0, \text { otro caso }\end{array}\right.$ \\
$Y_{k}^{r}=\left\{\begin{array}{l}1, \text { Si el EMM } k \text { es asignado a la ruta } r \in R \\
0, \text { otro caso }\end{array}\right.$ \\
$N_{j}^{r}=\left\{\begin{array}{l}1, \text { si la posición de almacenamiento } j \text { es visitada en la ruta } r \in R \\
0, \text { otro caso }\end{array}\right.$
\end{tabular}

Figura 3. Índices, parámetros y variables de decisión en el problema de ruteo de la preparación de pedido.

Fuente: elaboración propia. 


\section{Formulación del problema}

$$
\begin{gathered}
\operatorname{Min} T=\Sigma_{r \in R} \Sigma_{k \in K} \Sigma_{h \neq j \in L} \frac{d_{h j}}{V_{k}^{r}} \cdot S_{h j}^{r} \cdot Y_{k}^{r}+\Sigma_{r \in R} \Sigma_{k \in K} \Sigma_{i \in I} \Sigma_{j \in L} b_{i} \cdot t b e_{k i j} \cdot Y_{k}^{r} \cdot X_{i}^{r} \\
\sum_{i \in I} b_{i} \cdot L C_{i} \cdot X_{i}^{r} \leq A L C_{k} Y_{k}^{r} \forall r \in R, k \in K \\
\sum_{j \in L, j \neq h} S_{h j}^{r}=N_{h}^{r} \forall r \in R, h \in L \\
\sum_{h \in L, j \neq h} S_{h j}^{r}=N_{j}^{r} \forall r \in R, j \in L \\
\sum_{k \in K} \sum_{j \in L} Y_{k}^{r} N_{j}^{r}=\sum_{j \in L} N_{j}^{r} \forall r \in R \\
\sum_{h \in g, j \in L \backslash g} S_{h j}^{r} \geq N_{h}^{r} \forall r \in R, g \subseteq L \\
S_{h j}^{r}, X_{i}^{r}, N_{j}^{r} \varepsilon(0,1)
\end{gathered}
$$

La función objetivo (1) tiene como propósito minimizar el tiempo total para el conjunto de rutas de preparación de pedidos. La restricción (2) limita que la capacidad de carga del EMM $k$ no sea excedida por la cantidad de productos $i$ en una ruta $r R$. Las restricciones (3) y (4) velan por que cada posición de almacenamiento tenga un predecesor y sucesor en una ruta $r R$. La restricción (5) asegura que cada posición de almacenamiento $j$ asignada a una ruta $r R$ sea visitada por un EMM $k K$. La restricción (6) evita subrutas en un conjunto de $L$ posiciones de almacenamiento en el CEDI bajo modelamiento. La restricción (7), establece que las variables de decisión sean números binarios.

El problema de ruteo en la preparación de pedidos formulado puede ser solucionado con un algoritmo genético, búsqueda tabú clásico u otros metaheurísticos, los cuales permiten obtener soluciones pseudóptimas del menor tiempo posible. A partir de la revisión de la literatura y con las corridas experimentales ejecutadas, se justifica la selección de estos metaheurísticos, ya que: i) permiten resolver problemas de ruteo en CEDI con más de 40 posiciones de almacenamiento; ii) genera mejores soluciones que las reglas como el FLNL (Farhest Locations, Nearest Location) y heurísticos tales como: S-Shape, Largest Gap, Combinated, entre otros; iii) experimentos realizados con modelos de optimización fueron capaces de resolver problemas hasta de 45 posiciones aproximadamente, de allí la justificación del uso de los metaheurísticos [5, 8]. Finalmente, debe resaltarse que el ruteo de los equipos de manejo de materiales 
son críticos para aumentar la eficiencia energética que impacta directamente en la generación de emisiones $\mathrm{CO}_{2}$, lo cual justifica el modelamiento de este componente en el CEDI verde.

\section{RESULTADOS YDISCUSIÓN}

En esta sección se presenta la implementación del modelo de CEDI verde en una empresa mediana que comercializa alimentos en la ciudad de Medellín. La propuesta cubre los tres componentes del modelo que incluye: gestión del CEDI verde, TIC denominada $W M S$ y metaheurístico para resolver el problema de ruteo en la preparación de pedidos. La empresa en estudio es seleccionada porque: i) la dirección tiene interés en implementar políticas y prácticas verdes en sus procesos logísticos, ii) el CEDI tiene 200 posiciones de almacenamiento divididas en cuatro pasillos principales (figura 4), iii) la operación de preparación de pedidos es clasificada como crítica, ya que representa un $40 \%$ de los costos del CEDI y un alto impacto en el nivel de servicio al cliente y iv) la empresa se encuentra en proceso de implementación de un WMS, el cual es un desarrollo computacional propio que integrará a su ERP. Adicionalmente, en los resultados se incluye un análisis comparativo del estado actual y proyectado con el modelo de CEDI Verde (sección 2.1.3). A continuación, se presenta la distribución del CEDI en estudio (figura 4).

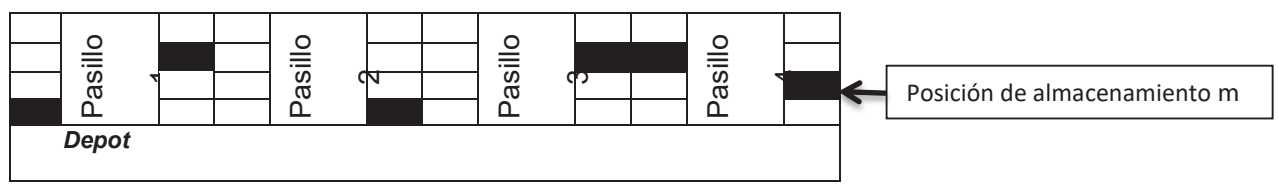

Nota: Depot es el lugar físico del CEDI donde inicia y terminan las rutas de acomodo y preparación de pedidos

Figura 4: configuración de CEDI de alimentos en estudio.

Fuente: elaboración propia

\subsection{Componente de gestión del modelo de CEDI verde}

En esta sección se presentan los resultados obtenidos de la implementación del componente de gestión del modelo de CEDI verde para la preparación de pedidos:

\subsubsection{Objetivos y estrategias en el modelo CEDI verde empresa de alimentos}

Los objetivos y estrategias diseñados en el modelo del CEDI verde se presentan abajo (tabla 3): 
Tabla 3: objetivos y estrategias en el modelo CEDI verde empresa de alimentos.

\begin{tabular}{|c|c|c|}
\hline $\begin{array}{l}\text { Objetivo } \\
\text { Verde }\end{array}$ & \multicolumn{2}{|c|}{$\begin{array}{l}\text { Ejecutar las operaciones en el CEDI utilizando recursos que generen la menor cantidad de } \\
\text { emisiones } \mathrm{CO}_{2} \text { posibles, promoviendo la política de un proceso sin papel, lo que permite } \\
\text { una logística amigable con el medio ambiente. }\end{array}$} \\
\hline \multirow{6}{*}{$\begin{array}{l}\text { Objetivo } \\
\text { específico } 1\end{array}$} & \multirow{6}{*}{$\begin{array}{l}\text { Reducir la can- } \\
\text { tidad de emisio- } \\
\text { nes de } \mathrm{CO}_{2} \text { en } \\
\text { el CEDI en al } \\
\text { menos un } 10 \%\end{array}$} & Estrategia 1.1 \\
\hline & & $\begin{array}{l}\text { Utilizar equipos de manejo de materiales eléctricos que generen bajas } \\
\text { emisiones de } \mathrm{CO}_{2} \text {. }\end{array}$ \\
\hline & & Estrategia 1.2 \\
\hline & & $\begin{array}{l}\text { Rediseñar las operaciones del CEDI, en especial el ruteo en la preparación } \\
\text { de pedidos, con el fin de reducir los metros recorridos por ruta, lo cual } \\
\text { disminuye la producción de } \mathrm{CO}_{2} \text {. }\end{array}$ \\
\hline & & Estrategia 1.3 \\
\hline & & $\begin{array}{l}\text { Implementar mejoras en la infraestructura del CEDI tales como: cambio } \\
\text { de sistemas de iluminación artificial por luz natural o paneles solares, } \\
\text { plan de manejo de agua, utilización de energía limpia para equipos de } \\
\text { manejo de carga, entre otros. }\end{array}$ \\
\hline \multirow[b]{2}{*}{$\begin{array}{l}\text { Objetivo } \\
\text { específico } 2\end{array}$} & \multirow{2}{*}{$\begin{array}{l}\text { Disminuir el } \\
\text { uso de papel } \\
\text { en al menos un } \\
80 \% \text {. }\end{array}$} & Estrategia 2.1 \\
\hline & & $\begin{array}{l}\text { Sistematizar las operaciones del CEDI utilizando el } W M S \text {. Únicamente } \\
\text { utilizar papel en actividades que lo exija la ley, por ejemplo, facturas y } \\
\text { documentos para el transporte. }\end{array}$ \\
\hline \multirow[b]{2}{*}{$\begin{array}{l}\text { Objetivo } \\
\text { específico } 3\end{array}$} & \multirow[b]{2}{*}{$\begin{array}{l}\text { Aumentar la } \\
\text { reutilización } \\
\text { del material de } \\
\text { embalaje (cajas } \\
\text { y estibas) en } \\
\text { mínimo un } 50 \%\end{array}$} & Estrategia 3.1 \\
\hline & & $\begin{array}{l}\text { Política de sincronización con los proveedores para que entreguen las } \\
\text { materias primas en cajas de cartón sin logos impresos que puedan ser } \\
\text { utilizados para empacar los productos a despachar por la empresa. Adi- } \\
\text { cionalmente, entrega de carga en estibas plásticas de medidas estándar de } \\
\text { 1x1.20 m que puedan ser reutilizadas en el almacenamiento o distribución. }\end{array}$ \\
\hline
\end{tabular}

\subsubsection{Caracterización de los recursos, operaciones y prácticas verdes en el CEDI}

A partir del análisis de la eficiencia, servicio al cliente, impactos en el medio ambiente (emisiones $\mathrm{CO}_{2}$ y consumo de papel) y costos de las operaciones del CEDI, se determinó que la preparación de pedidos es la más importante en el sistema logístico. Esto se plantea porque la operación representa el $40 \%$ de los costos del CEDI, tiene un impacto del 90\% en la satisfacción de los clientes (permite recoger los productos que conforman los pedidos respecto a referencias, cantidades y tiempos pactados), produce aproximadamente el 35\% de las emisiones de $\mathrm{CO}_{2}$ y utiliza un 55\% del papel del CEDI (órdenes o facturas de pedidos de los clientes). A continuación, se presenta la caracterización de la operación utilizando el Sipoc adaptado (tabla 4): 
Tabla 4: caracterización de la preparación de pedidos en el modelo de CEDI verde

\begin{tabular}{|c|c|c|c|c|c|c|}
\hline Operación & \multicolumn{2}{|l|}{ Preparación de pedidos } & Alcance & \multicolumn{3}{|c|}{$\begin{array}{l}\text { Cubre desde el procesamiento de las } \\
\text { órdenes de los clientes hasta su reco- } \\
\text { gida ejecutando las rutas configuradas } \\
\text { con los metaheurísticos. }\end{array}$} \\
\hline Objetivo & \multicolumn{2}{|c|}{$\begin{array}{l}\text { Recoger los pedidos de los clientes con } \\
\text { una confiabilidad de mínimo el } 99 \%\end{array}$} & $\begin{array}{l}\text { Objetivos } \\
\text { CEDI } \\
\text { verde }\end{array}$ & \multicolumn{3}{|c|}{$\begin{array}{l}\text { Reducir las emisiones de } \mathrm{CO}_{2} \text { en la } \\
\text { operación en al menos un } 10 \% \text {. } \\
\text { Disminuir el uso de papel en la pre- } \\
\text { paración de pedidos un } 99 \% \text {. }\end{array}$} \\
\hline $\begin{array}{l}\text { Proveedor } \\
\text { (Suppliers) }\end{array}$ & Entradas (Inputs) & \multicolumn{2}{|c|}{ Actividades (Processes) } & $\begin{array}{l}\text { Salidas } \\
\text { (Outputs) }\end{array}$ & \begin{tabular}{|l|} 
Clientes \\
(Costumers)
\end{tabular} & Recursos \\
\hline Clientes & Órdenes de los clientes & \multicolumn{2}{|c|}{$\begin{array}{l}\text { Procesar las órdenes de } \\
\text { los clientes }\end{array}$} & $\begin{array}{l}\text { Órdenes } \\
\text { procesadas }\end{array}$ & $\begin{array}{l}\text { Prepara- } \\
\text { ción de } \\
\text { pedidos } \\
\text { (interna) } \\
\end{array}$ & $W M S$ \\
\hline $\begin{array}{l}\text { Prepara- } \\
\text { ción de } \\
\text { pedidos } \\
\text { (interna) }\end{array}$ & Órdenes procesadas & \multicolumn{2}{|c|}{$\begin{array}{l}\text { Diseñar rutas de prepara- } \\
\text { ción con el menor tiempo } \\
\text { posible }\end{array}$} & $\begin{array}{l}\text { Progra- } \\
\text { mación } \\
\text { de rutas } \\
\text { utilizando } \\
\text { metaheu- } \\
\text { risticos }\end{array}$ & $\begin{array}{l}\text { Prepara- } \\
\text { ción de } \\
\text { pedidos } \\
\text { (interna) }\end{array}$ & $\begin{array}{l}\text { WMS y me- } \\
\text { taheuris- } \\
\text { ticos para } \\
\text { resolver } \\
\text { problema } \\
\text { de ruteo }\end{array}$ \\
\hline $\begin{array}{l}\text { Prepara- } \\
\text { ción de } \\
\text { pedidos } \\
\text { (interna) }\end{array}$ & Programación de rutas & $\begin{array}{l}\text { Recoger los } \\
\text { los pedidos c } \\
\text { según las rut }\end{array}$ & $\begin{array}{l}\text { roductos de } \\
\text { e los clientes } \\
\text { as diseñadas }\end{array}$ & $\begin{array}{l}\text { Informe } \\
\text { de pedidos } \\
\text { recogidos } \\
\text { en las rutas }\end{array}$ & $\begin{array}{l}\text { Prepara- } \\
\text { ción de } \\
\text { pedidos } \\
\text { (interna) }\end{array}$ & $\begin{array}{l}\text { - } W M S \\
\text { que } \\
\text { guía las } \\
\text { rutas. } \\
\text { - Monta- } \\
\text { cargas }\end{array}$ \\
\hline \multicolumn{7}{|c|}{ Prácticas verdes en la operación } \\
\hline \multicolumn{7}{|c|}{$\begin{array}{l}\text { - Manejo de órdenes e información sistematizada en el WMS que elimina el uso de papel. } \\
\text { - El problema de ruteo se resuelve con metaheuristicos, que permite obtener rutas de mínimo tiempo. } \\
\text { Menor tiempo, menor distancia por ruta, lo que reduce generación de kg de } \mathrm{CO}_{2} \text {. } \\
\text { - Infraestructura amigable con el medio ambiente: luz natural, energía limpia y utilización de montacar- } \\
\text { gas eléctricos. }\end{array}$} \\
\hline \multicolumn{7}{|c|}{ Recursos } \\
\hline Equipos & \multicolumn{6}{|c|}{$\begin{array}{l}\text { - Montacarga que utilizan energía eléctrica que producen bajas emisiones de } \mathrm{CO}_{2}(\mathrm{~kg}) \text {. } \\
\text { - Reemplazo de montacargas a gas por equipos eléctricos con generación de baja emisión } \\
\text { de } \mathrm{CO}_{2} \text {. }\end{array}$} \\
\hline Personal & \multicolumn{6}{|c|}{$\begin{array}{l}\text { - Entrenamiento al personal en el uso eficiente de recursos para reducir emisiones de } \\
\mathrm{CO}_{2} \text {. Esto incluye uso de la luz, el agua y otros recursos que se emplean en el CEDI. } \\
\text { - Entrenamiento en el uso del WMS como TIC para ejecutar las operaciones del } \\
\text { CEDI. }\end{array}$} \\
\hline
\end{tabular}




\begin{tabular}{|l|l|}
\hline Infraestructura & $\begin{array}{l}\text { - Instalación de tejas que permitan la entrada de luz natural y paneles solares para } \\
\text { energía limpia en el área de preparación de pedidos. } \\
\text { - Plan de sensibilización y control de certificados de revisión técnico-mecánica a los } \\
\text { vehículos que ingresan al CEDI. }\end{array}$ \\
\hline WMS & $\begin{array}{l}\text { - Esta TIC permite sistematizar las operaciones del CEDI, lo cual elimina el uso del } \\
\text { papel. } \\
\text { - Guía y control de la ejecución de las operaciones y recursos del CEDI usando } \\
\text { terminales móviles. }\end{array}$ \\
\hline $\begin{array}{l}\text { Material de } \\
\text { etiquetado }\end{array}$ & $\begin{array}{l}\text { - Las etiquetas son de papel ecológico y tintas amigables con el medio ambiente. } \\
\text { - Uso programado de etiquetas, es decir utilizar las necesarias. }\end{array}$ \\
\hline Indicadores del CEDI verde \\
\hline${ }^{*}$ Emisiones $\mathrm{CO}_{2}$ en kg en la preparación de pedidos \\
\hline${ }^{*}$ Cantidad de papel en la preparación de pedidos
\end{tabular}

Fuente: elaboración propia

Para calcular los valores actuales y proyectados de emisiones $\mathrm{CO}_{2}(\mathrm{~kg})$ en la operación de la preparación de pedidos en el CEDI, se utiliza una calculadora programada con la metodología para el cálculo de emisiones, dada por el IPCC (Panel Intergubernamental de Cambio Climático). A continuación, se listan las mediciones actuales de emisiones $\mathrm{CO}_{2}$ en $\mathrm{kg}$ y las cantidades de papel en kg utilizados en la operación en estudio (figura 5).

\begin{tabular}{|l|c|c|c|}
\hline \multicolumn{1}{|c|}{ Recurso } & Insumo & Unidad de medida & Valor actual recurso \\
\hline Montacarga (1000 rutas/mes) & GLP & lt GLP/mes & $641 \mathrm{lt} \mathrm{GLP/mes}$ \\
\hline 15 lámparas de 400 w para iluminar el CEDI & Electricidad & $\mathrm{KWh} / \mathrm{mes}$ & $1800 \mathrm{Kwh}$ \\
\hline $\begin{array}{l}\text { Electricidad para operar computadores, termina- } \\
\text { les y servidores del } W M S\end{array}$ & Electricidad & $\mathrm{KWh} / \mathrm{mes}$ & $100 \mathrm{Kwh}$ \\
\hline Consumo de papel & papel & $\mathrm{kg} / \mathrm{mes}$ & $80 \mathrm{~kg} / \mathrm{mes}$ \\
\hline $\begin{array}{l}\text { Desechos y productos dañados de la preparación } \\
\text { de pedidos }\end{array}$ & Productos & $\mathrm{kg} / \mathrm{mes}$ & $10 \mathrm{~kg} / \mathrm{mes}$ \\
\hline
\end{tabular}

Figura 5. Medición actual de $\mathrm{kg}$ de $\mathrm{CO}_{2}$ equivalente en el CEDI de alimentos en estudio.

Fuente: elaboración propia

A partir de la información obtenida de los recursos que se emplean en la preparación de pedidos en el CEDI, se calcula que las emisiones de $\mathrm{CO}_{2}$ equivalentes es de $1931 \mathrm{~kg}$. Debe resaltarse que esta medición incluyó el GLP utilizado por los montacargas y la electricidad para las lámparas, computadores y terminales que permiten la operación del $W M S$. 


\subsection{Componente WMS}

Este segundo componente del modelo del CEDI verde es una TIC $W M S$ que le permite la sistematización computacional de las operaciones buscando que estas se ejecuten efectivamente. El $W M S$ en la empresa en estudio permite reducir en $88 \%$ el consumo de papel en la preparación de pedidos, ya que se pasaría de utilizar 80 a $10 \mathrm{~kg} / \mathrm{mes}$. Los $10 \mathrm{~kg} / \mathrm{mes}$ en la preparación de pedidos corresponden a la impresión de etiquetas y códigos asociados a los productos y órdenes de los clientes. La cantidad de reducción de papel se obtuvo de una medición realizada en el CEDI de la empresa de alimentos. De esta manera, se demuestra que el $W M S$ contribuye a la implementación del enfoque de CEDI verde.

\subsection{Modelamiento cuantitativo del problema de ruteo en la preparación de pedidos considerando $K$ EMM het- erogéneos en la empresa de alimentos (componente 3)}

En la actualidad la empresa de alimentos bajo estudio utiliza una regla denominada PLPC (posición más lejana a posición más cercana al punto de inicio de la ruta de preparación de pedidos). Esta regla de ruteo PLPC organiza la secuencia de la ruta comenzando por la posición de almacenamiento que se encuentra más lejana a la posición inicial de la recogida de la preparación de pedidos (depot) hasta recolectar todos los productos pertenecientes a la orden o lista de preparación.

Una limitación que tiene el PLPC para el ruteo es que genera una única solución, ya que no realiza una exploración sistemática del vecindario de búsqueda sino que utiliza una regla de asignación que no modela de forma integrada restricciones asociadas a las características de los $K$ EMM heterogéneos, la disponibilidad del inventario, las posiciones de almacenamiento (ubicación y los productos a ser recogidos [7, 8]. Por lo expuesto, el problema de ruteo en la preparación de pedidos considerando K EMM heterogéneos se soluciona con un metaheurístico de búsqueda tabú, con el fin de obtener los menores tiempos posibles de la operación. La búsqueda tabú es seleccionada como metaheurístico, ya que ha demostrado su eficiencia y calidad de soluciones para resolver este tipo de problemas en la preparación. Adicionalmente, autores tales como: Henn [7] y Kulak et al. [8] también implementaron búsqueda tabú para resolver eficientemente este tipo problemas en el CEDI.

A continuación, se presenta la planeación de la experimentación y configuración de la regla PLPC y el metaheurístico de búsqueda tabú para solucionar el problema de ruteo (etapa i), (tabla 6). 


\section{Tabla 5: planeación de la experimentación y modelos cuantitativos en el problema de ruteo}

\section{Parámetros de la preparación de pedidos en el CEDI empresa de alimentos}

El CEDI en la preparación de pedidos cuenta con dos montacargas a gas con capacidad de 2 toneladas que tienen su respectivo operario asignado. Las actividades de la preparación de pedidos se realizan utilizando terminales que tienen instalado el $W M S$ que guían la ejecución de las rutas en tiempo real (recogida de los productos). Por lo cual, no se requiere el uso de papeles en la operación.

Pedidos seleccionados y posiciones de almacenamiento

Para ejecutar los experimentos, se selecciona una lista de pedidos conformada por cuatro órdenes de clientes típicas en el CEDI

\begin{tabular}{|l|c|c|}
\hline \multicolumn{1}{|c|}{ Referencia } & Cantidad & Posiciones de almacenamiento \\
\hline Caja x 12 unidades café instantáneo & 10 & $1,2,3$ y 4 \\
\hline Caja x 12 unidades bebida de chocolate & 8 & $48,49,50,51,52$ \\
\hline Caja x 8 unidades de aceite vegetal & 5 & $12,14,15$ y 16 \\
\hline Paquete x 12 unidades de pasabocas surtidos & 4 & 32,34 y 35 \\
\hline
\end{tabular}

\section{Regla PLPC}

- Número de iteraciones es 1 . Debe resaltarse que la regla ejecuta una sola iteración para calcular las rutas de preparación de pedidos, ya que no realiza búsqueda en vecindarios. La regla de ruteo PLPC se modela computacionalmente en Microsoft Excel ${ }^{\mathbb{B}}$.

\section{Metaheurístico de búsqueda tabú}

- Se modela un metaheurístico de búsqueda tabú clásico con movimientos de intercambio e inserción.

- Tamaño de lista tabú: 5. El tamaño de lista tabú, se obtuvo de la ejecución de corridas experimentales que permitieron establecer que este tamaño generaba las mejores soluciones para el problema.

- Número de iteraciones: 1000 corridas.

- El metaheurístico de búsqueda tabú empleado para resolver el problema fue calibrado por medio de 500 corridas. De estas corridas se verificó la capacidad del metaheurístico, no solo para solucionar el problema sino también para generar soluciones factibles.

- El metaheurístico de búsqueda tabú para solucionar el problema se ejecutó en un framework OpenTS que utiliza Netbeans, IDE 7.0.1, disponible para Windows ${ }^{\circledR}$ en un computador con procesador Intel Pentium 4 de $3.20 \mathrm{GHz}$ y memoria RAM de $1 \mathrm{~Gb}$.

Una vez se ejecuta la regla PLCP y el metaheurístico de búsqueda tabú para resolver el problema de ruteo en la preparación de pedidos, a continuación se presentan los siguientes resultados (Tabla 6: resultados de regla PLPC y búsqueda tabú para resolver problema de ruteo.

Fuente: elaboración propia:

Una vez se ejecuta la regla PLCP y el metaheurístico de búsqueda tabú para resolver el problema de ruteo en la preparación de pedidos, a continuación se presentan los siguientes resultados (tabla 7): 
Tabla 6: resultados de regla PLPC y búsqueda tabú para resolver problema de ruteo.

\begin{tabular}{|l|c|c|}
\hline & Regla PLPC & Búsqueda tabú \\
\hline Tiempo preparación de pedidos (min/ruta) & 15.40 & 12.50 \\
\hline Número promedio de rutas de preparación de pedidos por mes & 900 & 900 \\
\hline $\mathrm{CO}_{2}$ equivalente en $\mathrm{kg}$ & 1931 & 1733 \\
\hline Reducción del $\mathrm{CO}_{2}$ equivalente en $\mathrm{kg}$ & \multicolumn{2}{|c|}{198} \\
\hline Incremento en la eficiencia operacional/mes & \multicolumn{2}{|c|}{$18,83 \% / \mathrm{mes}$} \\
\hline
\end{tabular}

Nota: Debe tenerse en cuenta que los montacargas a gas son la fuente principal de CO2 en la operación.

Fuente: elaboración propia

A partir de los resultados obtenidos (tabla 7), se identifica que la implementación del metaheurístico de búsqueda tabú para resolver el problema de ruteo en la preparación de pedidos no solo incrementó la eficiencia operacional en un 18,83\% sino que también permite una reducción de $198 \mathrm{~kg} \mathrm{CO}_{2}$ equivalentes respecto a la regla PLPC. Una vez implementados los tres componentes del modelo CEDI verde en la empresa en estudio, a continuación, se presentan propuestas de otras prácticas verdes en este proceso logístico:

- En la actualidad el CEDI tiene instaladas 15 lámparas de 400 w. Estas se sustituirían por 10 lámparas de $250 \mathrm{~W}$ y se instalarían tejas translucidas.

- Sustitución de los montacargas de gas GLP por eléctricos, los cuales consumen aproximadamente $11 \mathrm{kWh}$ y para un ciclo de trabajo en el CEDI de 8 horas (duración de la batería) sería un consumo de $88 \mathrm{kWh}$.

Finalmente, se presentan los indicadores actuales y esperados con la implementación del modelo de CEDI (figura 6).

\begin{tabular}{|l|c|c|c|}
\hline \multicolumn{1}{|c|}{ Recurso } & Actual & Propuesto & Indicador \\
\hline Consumo GLP Montacarga* & 641 & 520 & lt GLP/mes \\
\hline Lámparas para iluminar el CEDI* & 1800 & 750 & $\mathrm{kWh} / \mathrm{mes}$ \\
\hline Electricidad para operar computadores, terminales y servidores del WMS & 100 & 100 & $\mathrm{kWh} / \mathrm{mes}$ \\
\hline Consumo de papel & 80 & 10 & $\mathrm{~kg} / \mathrm{mes}$ \\
\hline Material impreso de etiquetado & 20 & 20 & $\mathrm{~kg} / \mathrm{mes}$ \\
\hline Desechos y productos dañados de la preparación de pedidos & 10 & 10 & $\mathrm{~kg} / \mathrm{mes}$ \\
\hline Material de empaque y embalaje reutilizado & 100 & 100 & Unidades/mes \\
\hline
\end{tabular}

Figura 6: mediciones de indicadores del CEDI verde: actual y propuesto. 
A partir de la implementación de los tres componentes del modelo de CEDI verde, incluyendo la adopción de prácticas verdes respecto al cambio de las lámparas e instalación de tejas translucidas, se generan emisiones de $\mathrm{CO}_{2}$ equivalentes a 1205 kg. Por lo tanto, con el modelo de CEDI verde no solo se reducen las emisiones de $\mathrm{CO}_{2}$ equivalente en $731 \mathrm{~kg}$ o un $37 \%$ de las emisiones, ya que el valor actual calculado para el CEDI de alimentos es de $1933 \mathrm{~kg}$. Debe resaltarse que esta reducción se justifica principalmente en la implementación del metaheurístico de búsqueda tabú para resolver el problema de ruteo en la preparación de pedidos, que permitió una disminución de $198 \mathrm{~kg}$ de $\mathrm{CO}_{2}$ equivalente en el CEDI. Otra contribución significativa del modelo de CEDI verde se obtiene con la utilización del WMS, ya que permitió reducir el uso de papel en la preparación de pedidos en un $88 \%$, ya que se pasó de consumir 80 a $10 \mathrm{~kg} / \mathrm{mes}$. Esto se logró porque el $W M S$ sistematizó las actividades de esta operación del CEDI eliminando la necesidad del uso del papel.

\section{CONCLUSIONES}

La principal contribución del artículo fue el desarrollo y validación de un modelo de CEDI verde soportado en la implementación de prácticas de gestión, uso de un WMS (TIC) y un metaheurístico que permiten apoyar las decisiones de diseño o mejoramiento de sus operaciones, con el fin operar eficientemente el proceso y reducir la cantidad de $\mathrm{kg}$ de $\mathrm{CO}_{2}$ producido. De esta manera, se contribuyó al avance del conocimiento en el área de investigación de la logística verde, ya que este artículo es uno de los primeros en abordar el enfoque de CEDI Verde en el ámbito científico. La validación del modelo se realizó en el CEDI de una empresa comercializadora de alimentos en la ciudad de Medellín. Con la implementación del modelo de CEDI verde no solo la empresa ha establecido un componente de gestión verde sino que también redujo las emisiones $\mathrm{CO}_{2}$ equivalentes en $731 \mathrm{~kg}$ o un $37 \%$ de las emisiones, ya que pasó de un valor de $1933 \mathrm{~kg}$ por mes a $1205 \mathrm{~kg}$ por mes. Adicionalmente, con la implementación del $W M S$ y su integración en el modelo el CEDI verde, se disminuyó el uso del papel en un $88 \%$, lo que permitió reducir el consumo 80 a $10 \mathrm{~kg} / \mathrm{mes}$. Estos resultados permitieron validar las contribuciones del modelo del CEDI verde a la amigabilidad de este proceso logístico con el medio ambiente.

El componente de metaheurístico de búsqueda tabú para solucionar el problema de ruteo en la preparación de pedidos en el modelo de CEDI verde no solo permitió disminuir el tiempo promedio de cada ruta en aproximadamente 3 minutos y aumentar la eficiencia de la operación en un $18,83 \%$, sino también a obtener una reducción de $198 \mathrm{~kg} \mathrm{CO}_{2}$ equivalente, respecto a la utilización de la regla PLPC (estrategia de ruteo actual). De esta manera, se validó que este componente de modelamiento matemático del CEDI verde genera impactos positivos a la eficiencia y al medio ambiente. Finalmente, 
como trabajo futuro se plantea sincronizar otros procesos logísticos como transporte y logística inversa con el modelo de CEDI verde buscando contribuir a la reducción global de emisiones $\mathrm{CO}_{2}$ en el sistema logístico. Finalmente, también se detecta como oportunidad de investigación futura, identificar qué otros problemas cuantitativos en el CEDI se pueden formular, con el fin de contribuir a la disminución de emisiones $\mathrm{CO}_{2}$ y al aumento de la eficiencia del proceso logístico.

\section{REFERENCIAS}

[1] A. Correa and R. Gómez, “Tecnologías de la Información en la cadena de suministro,” Dyna, vol. 76, n. $^{\circ} 157$, pp. $37-48,2009$.

[2] E. Frazelle and R. Sojo, Logística de almacenamiento y manejo de materiales de clase mundial. Bogotá: Grupo Editorial Norma, 2007.

[3] J. J. Bartholdi and S. T. Hackman, Warehouse \& Distribution Science: Release 0.96. The Supply Chain and Logistics Institute, 2014.

[4] A. Correa, R. Gómez and J. Cano, "Gestión de almacenes y tecnologías de la información y comunicación (TIC),” Estudios Gerenciales, vol. 26, n. ${ }^{\circ}$ 117, pp. 145-171, 2010.

[5] S. Henn and V. Schmid, "Metaheuristics for order batching and sequencing in manual order picking systems," Computers \& Industrial Engineering, vol. 66, n. ${ }^{\circ}$ 2, pp. 338-351, Oct. 2013.

[6] H. Min, "Application of a decision support system to strategic warehousing decisions," International Journal of Physical Distribution \& Logistics Management, vol. 39, n. ${ }^{\circ}$ 4, pp. 270-281, 2009.

[7] S. Henn, "Metaheuristics for the order batching problem in manual order picking systems," BuR Business Research Journal, vol. 3, n. ${ }^{\circ}$ 1, pp. 1-24, 2010.

[8] O. Kulak, Y. Sahin, and M. E. Taner, "Joint order batching and picker routing in single and multiple-cross-aisle warehouses using cluster-based tabu search algorithms," Flex Serv Manuf $J$, vol. 24, n. ${ }^{\circ}$, pp. 52-80, Mar. 2012.

[9] M. de Brito and R. Dekker, "Reverse logistics - a framework," Econometric Institute Research Papers, vol. 1, pp. 1-19, Oct. 2002.

[10] S. Ubeda, F. J. Arcelus, and J. Faulin, “Green logistics at Eroski: A case study," International Journal of Production Economics, vol. 131, n. ${ }^{\circ}$ 1, pp. 44-51, May 2011.

[11] R. Dekker, J. Bloemhof, and I. Mallidis, "Operations Research for green logistics - An overview of aspects, issues, contributions and challenges," European Journal of Operational Research, vol. 219, n. 3 , pp. 671-679, Jun. 2012.

[12] U. Martinsen and M. Björklund, "Matches and gaps in the green logistics market," Int Jnl Phys Dist \& Log Manage, vol. 42, n. ${ }^{\circ}$ 6, pp. 562-583, Jul. 2012. 
[13] G. Zhang and Z. Zhao, "Green Packaging Management of Logistics Enterprises," Physics Procedia, vol. 24, Part B, pp. 900-905, 2012.

[14 ] Y. Y. Zhao and Y. J. Gu, "Green Logistics and Social Sustainable Development," in Advanced Materials Research, 2014, vol. 962, pp. 2180-2184.

[15 ] I. Harris, C. L. Mumford, and M. M. Naim, "A hybrid multi-objective approach to capacitated facility location with flexible store allocation for green logistics modeling," Transportation Research Part E: Logistics and Transportation Review, vol. 66, pp. 1-22, Jun. 2014.

[16] S. Zhang, C. K. M. Lee, H. K. Chan, K. L. Choy, and Z. Wu, "Swarm intelligence applied in green logistics: A literature review," Engineering Applications of Artificial Intelligence, vol. 37, pp. 154-169, Jan. 2015.

[17] K. Lai and C. W. Y. Wong, "Green logistics management and performance: Some empirical evidence from Chinese manufacturing exporters," Omega, vol. 40, n. ${ }^{\circ}$ 3, pp. 267-282, Jun. 2012.

[18] M. S. Pishvaee, S. A. Torabi, and J. Razmi, "Credibility-based fuzzy mathematical programming model for green logistics design under uncertainty," Computers \& Industrial Engineering, vol. 62, n. ${ }^{\circ}$ 2, pp. 624-632, Mar. 2012.

[19] K. Dobers, A. Klukas, W. Lammers, M. Laux, G. Mauer, and M. Schneider, "Green Logistics: Optimisation Approaches for Resource-Efficient Logistics Services," in Efficiency and Logistics, U. Clausen, M. ten Hompel, and M. Klumpp, Eds. Springer Berlin Heidelberg, 2013, pp. 149-161.

[20] G. Ćirović, D. Pamučar, and D. Božanić, “Green logistic vehicle routing problem: Routing light delivery vehicles in urban areas using a neuro-fuzzy model," Expert Systems with Applications, vol. 41, n. ${ }^{\circ}$ 9, pp. 4245-4258, Jul. 2014. 